\title{
POLÍTICAS E INICIATIVAS PARA EL ABORDAJE DE LAS PÉRDIDAS Y EL DESPERDICIO ALIMENTARIO EN ESPAÑA. RED SIN DESPERDICIO
}

\author{
Berta Vidal-Mones $^{\mathrm{a}}$, Héctor Barco ${ }^{\mathrm{b}}$, Aintzane Pérez-Ezkurdia ${ }^{\mathrm{c}}, \mathrm{M}^{\mathrm{a}} \mathrm{Jesús}_{\text {Casasnovas }}^{\mathrm{d}}$, Raquel Díaz- $^{-}$ \\ Ruíz $^{\mathrm{e}}$ y María Ángeles Fernández-Zamudio ${ }^{*}$
}

${ }^{a}$ CREDA-UPC-IRTA, (Castelldefels, Barcelona, berta.vidal@upc.edu). ${ }^{b}$ Enraíza Derechos, (San Sebastián, h.barco@enraizaderechos.org). 'Oficina de Prevención de Residuos y de Impulso a la Economía Circular (Navarra, aperezez@navarra.es). ${ }^{d} D . G$. de Protección de Consumidores y Usuarios. Dpto. de Ciudadanía y Derechos Sociales. Gobierno de Aragón (Zaragoza, mjcasasnovaso@aragon.es . ${ }^{e}$ Fundación Espigoladors (Barcelona, raquel@espigoladors.com). ${ }^{f}$ Instituto Valenciano de Investigaciones Agrarias. Centro para el Desarrollo de la Agricultura Sostenible (Moncada, Valencia, fernandez_marzam@gva.es).

\begin{abstract}
Resumen
En los Objetivos de Desarrollo Sostenible se recoge la necesidad de reducir las pérdidas y el desperdicio alimentario (PDA) en un 50\% para el año 2030. Desde la UE se insta a todos los Estados miembros a conocer sus cifras y a que realicen acciones que contribuyan a dicha meta. En España, cada Comunidad Autónoma está implantando su propia agenda y los enfoques son múltiples. En una primera parte del presente trabajo se repasan y se valoran las principales políticas regionales que están realizándose para el abordaje de las PDA. Se comprueba que las iniciativas sobre las PDA las están desarrollando numerosas personas que trabajan en diversas administraciones y entidades. La evidente necesidad de avanzar en red ha incentivado la creación de la Red Sin Desperdicio, un foro en el que se buscan sinergias e intercambio de aprendizajes colectivos. En su primera iniciativa conjunta se realizó un sondeo durante el primer gran confinamiento por COVID-19, y se recabó información de más de 6.250 hogares. Se muestran los principales resultados del comportamiento que han tenido las familias.
\end{abstract}

Palabras clave: hogares, gestión de alimentos, percepción del desperdicio, consumo responsable, políticas alimentarias.

\section{Introducción y objetivos}

Producir la comida exige un elevado consumo de recursos (tierra, agua, mano de obra, químicos, maquinaria o energía), si al final los alimentos se tiran a la basura estos recursos no habrán servido para nada y se habrán generado en vano importantes impactos sociales, económicos y ambientales. Para ir enfrentando este grave problema, la Comisión Europea incluyó en 2015 el desperdicio alimentario (DA) en el "Plan de acción para la economía circular"; también en ese año las Naciones Unidas contemplaron su abordaje, concretamente dentro de los ODS, y en el sub-objetivo 12.3 se plantea la meta de reducir los actuales niveles de DA a la mitad para 2030.

En los últimos años la UE ha seguido desarrollando distintas normativas, con ellas se insta a los Estados miembros a asumir un rol mucho más activo en la lucha contra el DA. En España esto a su vez se traduce en que las comunidades autónomas (CCAA) vayan impulsando sus propias agendas. Deducir cuales son las políticas institucionales que se desarrollan en España es el primer objetivo del presente trabajo.

Por otra parte, aunque se producen PDA en todos los eslabones de la cadena agroalimentaria, donde más se genera es en el consumo final, particularmente en los hogares. Todo ello se acentúa en un contexto como el que supuso el primer gran confinamiento por la COVID-19, y fue este el momento en que un grupo de profesionales del sector agroalimentario y ambiental decidieron crear la "Red Sin Desperdicio" para contribuir al conocimiento y prevención de las PDA. El segundo objetivo del presente trabajo es presentar los principales resultados de su primer trabajo conjunto, un sondeo para analizar cómo estaba siendo la gestión de los alimentos en los hogares españoles durante ese confinamiento, y cuál era la percepción que tenían los hogares españoles sobre la generación de DA.

\section{Metodología}

Respecto al primer objetivo se ha realizado una revisión y puesta en común de las normas e iniciativas que están usando para luchar contra las PDA tanto el gobierno de España como las Autonomías.

Respecto al sondeo a hogares, se realizó del 14 de mayo al 11 de junio de 2020, cuando la movilidad de la ciudadanía española estuvo muy restringida. El cuestionario tenía 36 preguntas y se distribuyó por redes sociales y correos masivos. Se trata por lo tanto de un muestreo no probabilístico y de conveniencia. Tras revisar las respuestas recibidas se agruparon un total de 6.293 respuestas válidas. La procedencia de las 6.293 respuestas fue: 48\% Aragón, 13\% Cataluña, 13\% Comunitat Valenciana, 8\% Navarra, 7\% Andalucía, 
$4 \%$ País Vasco, 4\% Madrid, y el 4\% restante (que suma 269 respuestas) del resto de comunidades autónomas. El perfil medio de las personas que respondieron es este: en su mayoría eran mujeres $(74,6 \%$ de la muestra), de edad entre 41 y 60 años $(60,1 \%)$, solo el $34 \%$ de los hogares tenían menores de 14 años, y en un $68,6 \%$ manifestaron que estaban trabajando. De forma mayoritaria respondieron personas que tenían un papel activo y cotidiano tanto en la compra de alimentos como en la elaboración de la comida en los hogares, lo que les hace muy conocedoras de todo lo que se preguntaba.

La muestra fue caracterizada a través de un análisis descriptivo. Se realizaron análisis de varianza (T-tests y ANOVA) para valorar diferencias apreciables entre los grupos. Se analizó si existían diferencias significativas en las cifras de desperdicio alimentario por hogar y per cápita, diferenciando por grupos de edad, género, número de miembros por hogar, hogares con menores, o por la situación laboral. Se evaluaron los posibles cambios en los hábitos alimentarios y de compras de alimentos durante la pandemia. También se hicieron test de tipo post hoc (tests de Games-Howell) para valorar qué categorías difieren significativamente unas de otras en relación a las cifras de desperdicio alimentario por hogar y per cápita, aplicándolos al caso en que las variables tuvieran más de 2 categorías de respuestas.

\section{Resultados}

\subsection{Sobre las políticas e iniciativas con las que se está abordando las PDA en España}

A nivel de Estado español existe la Estrategia "Más alimento, menos desperdicio" que lanzó el Ministerio de Agricultura, Pesca y Alimentación (MAPA) en 2013, la Estrategia que está ahora en vigor es la del periodo 2017-2020. El MAPA ha promovido estudios puntuales en los diferentes eslabones de la cadena, participa en numerosos actos para dar visualización al problema, además de apoyar diferentes iniciativas realizadas por entidades públicas y privadas. Se considera especialmente interesante el "Panel de cuantificación del DA en los hogares", que ha desarrollado tomando como base la amplia cobertura de datos que ofrece el "Panel de consumo alimentario", lo que está permitiendo tener una estimación aproximada del nivel de DA que se produce en los hogares españoles desde 2015.

Recientemente el MAPA anunció el desarrollo de una ley estatal sobre las PDA en los próximos meses, pero al margen de la misma existen distintas iniciativas legislativas que contemplan colateralmente el abordaje del desperdicio de alimentos. Se pone como ejemplo el Código de Buenas Prácticas en la Contratación Alimentaria, encuadrado en la Ley 12/2013, de 2 de agosto de medidas para mejorar el funcionamiento de la Cadena Alimentaria, que podría tener un importante impacto si se cumpliera, aunque al ser de adhesión voluntaria, de momento su repercusión en la reducción del DA es escasa.

Respecto a la actuación de las distintas CCAA, se observa gran disparidad de circunstancias. También es muy variable el sentido que se da a los trabajos, así en algunas administraciones el abordaje de las PDA se focaliza en los departamentos de agricultura y alimentación, en otras en el área de residuos, en otras en consumo, etc. En general los trabajos específicos para conocer la envergadura del problema del DA en los territorios y las actuaciones globales para su minimización, son aún muy puntuales en la mayoría de las CCAA. También son limitados los recursos materiales y humanos empleados en enfrentar este problema, por lo que tendrán que intensificarse los mismos si se pretende cubrir la meta marcada con los ODS, así como los planteamientos derivados de la Decisión Delegada 2019/1597 de 3 de mayo de 2019, con la que la UE insta a los Estados miembros a la cuantificación del DA, y a comunicar dichas cifras de manera periódica. En la práctica cada vez es más frecuente la introducción de la agenda del desperdicio alimentario en otros temas transversales, como las estrategias para luchar contra el cambio climático, el alcance de la transición ecológica, la extensión de la economía circular, o la mejora del funcionamiento de la cadena alimentaria, etc., ya que en todas ellas hay cuestiones comunes con la reducción de las PDA.

Entre las CCAA Cataluña es la que tiene una experiencia más amplia de trabajo con las PDA, de hecho diseñó su primer Plan de Residuos Municipales en el periodo 1995-2000. Se podría decir que siempre han estado en la vanguardia y a las muchas iniciativas surgidas por su sociedad civil, se une el trabajo realizado en organismos de investigación y universidades. Por ejemplo el CREDA participó en el proyecto europeo REFRESH, que junto FUSIONS son los dos proyectos de investigación que han abordado de manera más rigurosa y amplia el problema de las PDA. Hasta el momento Cataluña ha sido la única en desarrollar una ley propia (Llei 3/2020, de l'11 de març, de prevenció de les pèrdues i el malbaratament alimentaris). Con ella se derivan obligaciones para todos los agentes de la cadena, y se regulan las correspondientes sanciones por incumplimientos. Las empresas que distribuyen alimentos deberán tener un plan de prevención de las PDA, y deberán medirlas e informar de ellas. Para ayudar a este reto la administración catalana ha desarrollado la "Guía para la implantación de un plan de prevención y reducción de las PDA en las empresas agroalimentarias".

El resto de CCAA tienen una experiencia más limitada y reciente. En todos los territorios se insiste en que el trabajo debe ser con una visión intersectorial y están estableciendo agendas que implican a la ciudadanía. 
Como planes institucionales más globales están los del País Vasco (Plan de actuaciones contra el despilfarro de alimentos en Euskadi), la Comunitat Valenciana (Plan de acción contra el desperdicio alimentario en la Comunitat Valenciana, Plan BonProfit) o la Estrategia Sin Desperdicio 2030 de Castilla la Mancha. Por su parte en Navarra los trabajos sobre DA se enmarcan en el "Plan de residuos de Navarra 2017-2027", siguiendo sus actuaciones una perspectiva desde la gestión de los residuos.

Entre el resto de CCAA destaca Aragón, su DG de Consumo es muy activa en cuestiones de sensibilización. También han desarrollado el "Código Aragonés de buenas prácticas frente al desperdicio alimentario", que si bien es voluntario, son las propias empresas las que se comprometen en realizar buenas prácticas, algo que a su vez trasladan a la sociedad. Se menciona además a Cantabria, su "Estrategia contra el despilfarro de alimentos 2019-2022" hace hincapié en la emergencia social y la responsabilidad social de las empresas. En otras CCAA las iniciativas o son muy incipientes y aún no son suficientemente conocidas, o todavía son muy limitadas, o bien son inexistentes.

Revisando estos planes y estrategias se observa que el aspecto en que más se está incidiendo es el de sensibilización, hay un claro consenso en que falta concienciación algo clave para impulsar la prevención. Hay ámbitos donde todas ellas suelen trabajar, por ejemplo con los colegios. Por contra son muy pocas las que están midiendo las pérdidas de cosecha en el eslabón del origen, o que trabajan de forma específica con canales como HORECA, o que impulsan medidas novedosas para reforzar el trabajo que realizan entidades sociales, como los bancos de alimentos. Lo que sí se visualiza es interés en que el DA forme parte de los requerimientos que se hace en la licitación y compra pública de alimentos, y hay ayuntamientos de grandes ciudades, y algunos gobiernos autonómicos, que ya progresan en esto.

\subsection{Sondeo a hogares españoles durante el confinamiento por COVID-19}

En un primer bloque de preguntas se incidía sobre el comportamiento de los hogares, comparando el momento del confinamiento por COVID-19 respecto a lo que hacían previo a la pandemia. En la mayoría de las cuestiones la población señaló no haber modificado sus rutinas sobre la gestión de los alimentos. En relación a si habían comido más de lo habitual en ese periodo, el $24 \%$ del total reconoció que sí. Sobre si habían comprado más por ansiedad, de media solo el $31 \%$ dijo que sí, aunque con un valor muy desigual entre CCAA. Parece que hubo más consenso con el hecho de almacenar más comida de lo normal, dijo que sí un $45 \%$. También de forma muy global (el 75\%) dijeron haber salido menos a comprar, algo lógico por las limitaciones de movimiento que suponía el confinamiento. Al preguntar dónde compraron en este periodo, se comprueba que el 59\% indicaba que iban con más frecuencia a tiendas de barrio, algo que se superaba por ejemplo en Aragón, Cataluña, Comunitat Valenciana o País Vasco. La compra online también parece haber crecido en este periodo, se sabía que el comercio electrónico de alimentos era muy bajo en España, pero el 13\% indicó haber comprado alimentos online, un porcentaje que se supera en Cataluña y Madrid. Finalmente, a la pregunta de si desperdiciaron más en el confinamiento, hay una respuesta negativa muy unánime en todas las CCAA, una media del 96\%. En otro bloque se analizaron una serie de prácticas habituales en los hogares y se trataba de comprobar los cambios respecto al período pre-COVID-19, las respuestas se muestran en la Gráfica 1.

Gráfico 1. Comportamiento en la gestión de alimentos de los hogares durante el confinamiento

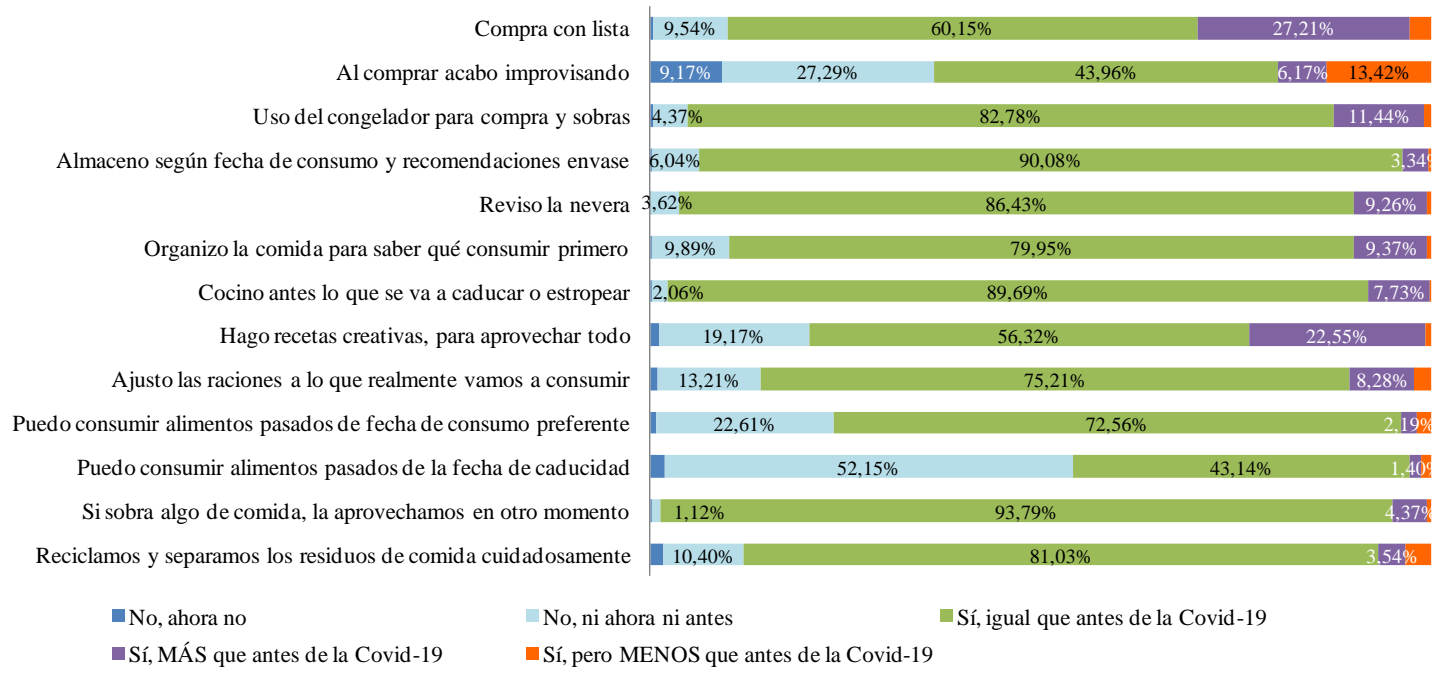


Se preguntó por el volumen de comida que tiraban, pero un gran número de hogares no sabían indicar una cifra, cuando lo hacen el $22 \%$ dice no tirar nada. Es muy uniforme la sensación de que en el hogar propio se desperdicia menos que en el resto de hogares (un 38\% dice tirar mucho menos que los demás).

En el Cuadro 1, se muestra cómo existe una relación significativa entre la edad y las cifras aportadas del DA tanto por hogar como per cápita, ambas cifras suele aumentar con la edad, una tendencia que cambia en los mayores de 60 años y los más jóvenes, que afirman desperdiciar considerablemente menos. El género también es otro de los parámetros significativos, los hombres reportan niveles superiores a las mujeres. Además, según aumenta el tamaño de la familia el DA es mayor, pero este parámetro se invierte si el DA se mide per cápita, a más personas en el hogar, menos DA per cápita. La situación laboral también es significativa en ambas cifras del DA, siendo mayores entre las personas en ERTE, seguidas por quienes teletrabajaban o trabajaban presencialmente. Las cifras más bajas es entre los encuestados cobran una pensión por jubilación, junto con quienes estaban en situación de desempleo.

Cuadro 1. Resumen de variables que muestran significación repecto al desperdicio en hogares

\begin{tabular}{|c|c|c|c|c|c|c|c|c|}
\hline \multirow[b]{3}{*}{ Variables } & \multirow[b]{3}{*}{ Variables y Categorías } & \multirow[b]{3}{*}{$\mathbf{N}$} & \multicolumn{3}{|c|}{ DA en hogares } & \multicolumn{3}{|c|}{ DA per capita } \\
\hline & & & \multicolumn{2}{|l|}{ Media } & & \multicolumn{2}{|l|}{ Media } & \multirow[b]{2}{*}{$p$-Valor } \\
\hline & & & (g) & $\mathrm{SD}(\mathrm{g})$ & $p$-valor & (g) & $\mathrm{SD}(\mathrm{g})$ & \\
\hline Total & & 3663 & 234.72 & 420.28 & & 88.42 & 154.57 & \\
\hline \multirow{5}{*}{ Edad } & $18-25$ & 50 & 149.58 & 209.88 & \multirow{5}{*}{0.000} & 58.28 & 75.98 & \multirow{5}{*}{0.000} \\
\hline & $26-35$ & 413 & 227.97 & 312.83 & & 111.65 & 164.20 & \\
\hline & $36-50$ & 1465 & 271.55 & 505.25 & & 91.02 & 159.45 & \\
\hline & $51-65$ & 1560 & 218.97 & 376.53 & & 84.37 & 154.58 & \\
\hline & Más de 65 & 175 & 107.05 & 151.41 & & 56.51 & 81.18 & \\
\hline \multirow{3}{*}{ Género } & Mujer & 2709 & 213.46 & 380.13 & \multirow{3}{*}{0.000} & 80.66 & 143.47 & \multirow{3}{*}{0.000} \\
\hline & Hombre & 929 & 294.40 & 514.81 & & 109.83 & 181.61 & \\
\hline & No declarado & 15 & 206.67 & 285.75 & & 96.22 & 105.65 & \\
\hline \multirow{6}{*}{$\begin{array}{l}\text { Situación } \\
\text { laboral durante } \\
\text { COVID-19 }\end{array}$} & Teletrabajando & 1483 & 261.97 & 498.24 & \multirow{6}{*}{0.001} & 95.03 & 168.70 & \multirow{6}{*}{0.034} \\
\hline & Trabajo en un sitio físico & 1133 & 230.36 & 328.75 & & 90.36 & 147.30 & \\
\hline & Sin empleo ni ingresos & 149 & 190.41 & 449.19 & & 64.81 & 121.43 & \\
\hline & Con prestaciones sociales por COVID-19 & 151 & 285.84 & 596.24 & & 95.63 & 156.64 & \\
\hline & Jubilación o pensionista & 458 & 177.71 & 332.12 & & 77.68 & 157.88 & \\
\hline & Otras situaciones & 289 & 198.46 & 259.09 & & 72.32 & 105.61 & \\
\hline
\end{tabular}

NOTA: $\mathrm{DA}=$ Desperdicio alimentario. $\mathrm{SD}=$ Desviación estándar. Las variables con más de 2 categorías se analizaron mediante ANOVA, mientras

que las variables con 2 categorías se analizaron mediante pruebas T-test. La diferencia de medias es significativa con un valor del p-valor $<0,05$

\section{Discusión y Conclusiones}

Revisando las iniciativas que se están poniendo en marcha en España para abordar el problema del desperdicio alimentario, se deduce que aún hay muchos territorios donde no existen agendas claras al respecto. Aunque los medios empleados en las CCAA para este fin son limitados, se intuye que esto podrá cambiar según sean mayores las presiones desde la UE para tener datos realistas de las cifras de DA. Conocer cuánto se desperdicia, los motivos de ello y dónde se producen estas pérdidas, será clave en los próximos años. Parece prioritario seguir dando visibilidad al problema, concienciar a la población en general, y a los agentes de la cadena agroalimentaria en particular, para que enfrenten el reto de reducir las PDA a corto plazo, al fin y al cabo el desperdicio es la evidencia de las fuertes ineficiencias que existen en la cadena, y su mejora la clave para que el sector siga siendo viable.

Respecto al sondeo realizado en los hogares españoles durante el confinamiento en general la población indica que no ha modificado sustancialmente sus hábitos, aunque sí se detectan ciertos cambios. Las dificultades para salir parece que fomentó el hacer compras más planificadas y a optimizar el almacenamiento y la conservación de los alimentos en casa, en la práctica todos estos son aspectos que conducen a un consumo más responsable. Otras prácticas favorables que se vieron incrementadas en este momento de la pandemia fue, utilizar más las listas de compra, o el realizar más recetas de aprovechamiento. Por otra parte el que la población compre más en tiendas de barrio, o se demanden más alimentos online, pueden abrir una oportunidad para la venta directa y los intermediarios, y con ello beneficiar a los productores, incrementando así su captación de valor dentro de la cadena agroalimentaria.

Sería deseable que estos comportamientos favorables se mantuvieran en el tiempo, pero el consumo suele ser imprevisible y se necesitarán otros estudios futuros. Contribuir a la prevención y reducción del DA pasa por dar visibilidad a este problema y sensibilizar a la población, la cual muestra un cierto grado de conciencia sobre el mismo pero no se siente responsable de ello, al pensar que es algo que originan otras personas. La clave parece estar en poner números al DA y fomentar acciones de participación colectiva.

\section{Agradecimientos}

Las autoras agradecen el apoyo de sus entidades y organismos. También al "Plan contra el desperdicio alimentario en la Comunitat Valenciana, Plan BonProfit” (con fondos FEDER), por el soporte dado. 